\title{
Toll-like receptor responses are suppressed in trauma ICU patients
}

Travis L. Holloway, MD ${ }^{1}$, Susannah E. Nicholson, $\mathrm{MD}^{1}$, Meenakshi Rani, $\mathrm{PhD}^{1}$, Andrew P. Cap, MD, PhD ${ }^{2,1}$, Martin G. Schwacha, $\mathrm{PhD}^{1,2}$

${ }^{1}$ The University of Texas Health Science Center at San Antonio, TX 78229, ${ }^{2}$ US Army

Institute of Surgical Research, JBSA Fort Sam Houston, TX 78234

\section{Corresponding Author:}

Martin G. Schwacha, $\mathrm{PhD}$

Dept of Surgery, The University of Texas Health Science Center at San Antonio, 7703 Floyd Curl Dr., San Antonio, TX 78229

Phone: 210-567-2187

FAX: 210-567-3447

Email: schwacha@uthscsa.edu

Author contributions: TLH modified study design, prepared compliance documents, collected and analyzed samples, prepared the initial draft of the manuscript and its revision and conducted all statistical analysis. SEN assisted in data interpretation, preparation of the initial draft of the manuscript and its revision. MR assisted in study design, sample analysis and manuscript revision. APC assisted with data interpretation and revised the manuscript. MGS was the principal investigator and oversaw all aspects of the study including design, interpretation of results, statistical analysis, as well as manuscript preparation. The authors declare that they have no competing interests. All authors read and approved the final version of the manuscript.

Running Head: TLRs and Trauma 


\section{ABSTRACT}

Background: Inflammation and activation of the innate immune system is often associated with traumatic injury and may involve alterations in Toll-like receptor (TLR)mediated responses.

Methods: A prospective observational study was designed and conducted. Twenty-one severely injured (ISS = 16-41) trauma ICU patients and 6 healthy volunteers that served as controls were enrolled. Anticoagulated whole blood was collected at 2-12 days after ICU admission and incubated in the presence of media alone (baseline), zymosan (TLR2 agonist) or LPS (TLR4 agonist) for $3 \mathrm{hrs.} \mathrm{Supernatant} \mathrm{levels} \mathrm{of} \mathrm{inflammatory} \mathrm{cytokines}$ (IL-1 $\beta,-6,-10$, TNF $\alpha$ ) were determined.

Results: TLR2 and TLR4-mediated activation of whole blood cell cultures from both healthy volunteers and subjects induced elevated cytokine levels over that observed in unstimulated cultures. Baseline values of IL-6 were significantly elevated in subject cultures as compared to healthy volunteers. Healthy volunteer cultures had 2-3-fold greater levels of IL-6 and TNF $\alpha$ than subject cultures when stimulated with zymosan (TLR2 agonist) or LPS (TLR4 agonist). IL-1 $\beta$ and IL-10 levels did not differ significantly between healthy volunteers and subjects.

Conclusions: The ability of circulating leukocytes from trauma ICU patients to be activated by TLR agonists is markedly suppressed and may play a role in the development of subsequent infectious complications.

Key words: Cytokines, Inflammation, Whole Blood, PAMPs, DAMPS 


\section{INTRODUCTION}

Trauma creates a significant medical and socioeconomic burden worldwide. Patients requiring mechanical ventilation, multiple operations, or a prolonged length of stay (LOS) in the intensive care unit (ICU) incur even greater costs [1-3]. Severe injury is associated with profound inflammation and can activate the innate immune system $[4 ; 5]$. The innate immune system is the first line of immunologic defense and its components are positioned at the interface between the external and internal environment. The innate response is immediate and non-specific; however, it lacks lasting immunologic memory. This approach is designed to allow for a quick defense against non-self antigens until longer-term immunity has an opportunity to mature.

Innate immune cells express pattern recognition receptors (PRRs) including toll-like receptors (TLRs) that are able to recognize non-self antigens [6;7]. Bacteria possess pathogen-associated molecular patterns (PAMPs) that can interact with TLRs and induce the innate immune response [8;9]. Bacterial lipopolysaccharide (LPS) is one such PAMP that is present in microbial cells, but not host cells. Recent studies have provided evidence that some host-derived molecules (also known as damage-associated molecular patterns; DAMPs) may also stimulate TLR2- or TLR4-dependent signaling [10;11]. DAMPs originate from a variety of cellular components including the plasma membrane, nucleus, endoplasmic reticulum, cytosol, and mitochondria [12]. This provides a method for which the body may immediately respond to widespread "sterile" injury that is not infectious in origin. This elicited response may be pro-inflammatory and/or antiinflammatory with the production of inflammatory mediators such as cytokines. TLR- 
mediated responses appear to be central to orchestrating the body's reaction to tissue damage as well as to pathogens. Alterations to these TLR-mediated responses may play a role in clinical outcome following injury $[10 ; 13]$. If the response to PAMPs is altered, it follows that the similar TLR-mediated response to DAMPs may also be affected.

Toll-like receptors are classified according to the types of agonists that bind and the corresponding response that is activated [14]. TLR2 and TLR4 are two separate TLRs that respond to differing signals. TLR2 mediates the host-response to gram positive bacteria and yeast. In addition to these exogenous signals, TLR2 also binds to endogenous agonists including heat-shock proteins (HSPs), HMGB1, and necrotic cells $[15 ; 16]$. In the laboratory, a commonly utilized TLR2 agonist is lipoteichoic acid (zymosan). TLR4 mediates the host-response to gram negative bacteria. LPS is the cell wall component of gram negative cells to which TLR4 binds, and it is also commercially available and acts as a TLR4 agonist. TLR4 also binds endogenous agonists including HSPs, HMGB1, surfactant protein A, and oxidized LDL. In this regard, activation of TLRs by corresponding known bacterial agonists could indicate reactivity with comparable DAMPs.

Since injury is often associated with a profound inflammatory response and activation of the innate immune system [4;5], we hypothesized that alterations in TLRs are instrumental in mediating this response. We conducted a prospective, observational study in severely injured trauma patients to evaluate the TLR-mediated inflammatory response. 
The goal of the study was to determine if severely injured patients had different TLRmediated responses than non-injured healthy individuals. 


\section{MATERIALS AND METHODS}

Subjects and Data Collection: A prospective observational trial was designed to enroll subjects from the surgical and trauma intensive care unit (STICU) that had severe traumatic injury. This study was conducted over a 1-year period and informed consent was obtained from patients and/or their legally authorized representative. Approval for this project was obtained from the University Hospital and The University of Texas Health Science Center at San Antonio Institutional Review Boards. A total of 21 severely injured subjects were enrolled and compared to six healthy volunteers. All of these subjects were admitted to the surgical ICU and all had been intubated and were on mechanical ventilation secondary to their injuries. Severe traumatic injury for this study was defined as at least one body region injury defined with an Abbreviated Injury Score (AIS) of 3 or greater. Traumatic brain injury (TBI) is known to cause immune dysregulation independent of other injury types; therefore TBI was not an inclusion criterion alone [17]. Inclusion injuries are shown in Table 1. Exclusion criteria included only those patients who fell within protected populations: those that were pregnant, less than 18 years of age, or prisoners. The subjects were enrolled and informed consent was obtained at the time of sample collection. Other patient information was captured in the following categories: demographics, injury severity scoring (ISS), injury mechanism, length of stay, mortality, and outcomes. For comparison, 6 normal volunteers also provided a blood sample for analysis of their systemic cytokine levels. The data presented herein are from the same subject group in a previous publication on acute lung injury[18]. 
Blood Collection and Processing: Subjects were enrolled between 2 and 12 days after ICU admission. A blood sample of approximately $6 \mathrm{~mL}$ was collected in an EDTA or heparin tube and processed within 1 hour. For comparison, 6 healthy volunteers were also enrolled and served as controls. A similar consent process was used for the control individuals. The control whole blood samples were obtained in an identical fashion to the experimental whole blood samples. A plasma sample was also collected and frozen for cytokine analysis.

In vitro TLR stimulation assay: The whole blood stimulation assay was used [19]. In brief, $1 \mathrm{~mL}$ of anticoagulated blood was added to a $15 \mathrm{~mL}$ Falcon tube. The blood was stimulated with zymosan $(33 \mu \mathrm{g} / \mathrm{mL})$ to activate TLR2, LPS $(10 \mathrm{ng} / \mathrm{mL})$ to activate TLR4 or left unstimulated. TLR agonists were obtained from Sigma Chemical (Zymosan A from Saccharomyces cerrevisiae) and Calbiochem (Lipopolysaccharide LPS, Ultra Pure, Salmonella minnesota R595). The agonist concentrations used were previously determined to be optimal for the induction of cytokine production [20]. The whole blood cultures were incubated at $37^{\circ} \mathrm{C}, 5 \% \mathrm{CO}_{2}$ with shaking for $3 \mathrm{hrs}$. Cell-free supernatants were collected and stored at $-80^{\circ} \mathrm{C}$ prior to cytokine determination.

Cytokine Analysis: Representative inflammatory cytokine levels (IL-1 $\beta,-6$, and TNF $\alpha$ ) and representative anti-inflammatory cytokine levels (IL-10) in the supernatants and plasma were measured using the Bio-Plex ${ }^{\mathrm{TM}}$ immunoassay (Bio-Rad Laboratories). 
Sample preparation, assay procedure, and analysis were performed according to the manufacturer's recommendations.

Data and Statistical Analysis: All data were handled confidentially and results were stored in a secure database. The data were maintained in accordance with institutional policy and access to the data was limited to the research team. Protected health information (PHI) was deleted from the database prior to final study analysis. The data are expressed as mean \pm SEM, unless noted otherwise. Comparisons were analyzed using ANOVA and SigmaPlot 11.0 software (Systat Software Inc). A $p$-value $<0.05$ was considered to be statistically significant for all analyses. 


\section{RESULTS}

Demographics: The enrolled subjects were representative of entire trauma patient population. The mean age of subjects was $40.7 \pm 19.7$ years of age (mean $\pm \mathrm{SD}$ ). Eighty-six percent of the study population was male. Ethnicity of the subjects mirrored that of the general population in south Texas, $43 \%$ were identified as Hispanic, while $57 \%$ were identified as Caucasian. Six of the subjects had measurable levels of alcohol in their blood at the time admission to the Emergency Department with a mean of $0.13 \pm$ $0.09 \%$. Of these subjects, four were legally intoxicated with a blood alcohol level > $0.08 \%$. All of the patients were severely injured, the mean ISS was $31 \pm 11$ (mean $\pm \mathrm{SD}$ ). The average ICU length of stay (LOS) prior to enrollment and sample collection was 6.6 \pm 4.2 days (mean \pm SD). Overall, the subjects had a mean ICU LOS of 20.6 days with a mean hospital LOS of 37.6 days. These demographic data are shown in Table 2.

Plasma Cytokine Levels: Levels of TNF $\alpha$, IL-1 $\beta$, IL-6, and IL-10 were measured in plasma samples from both subjects and controls. Only IL-6 levels in the subjects showed a significant elevation over that of controls or normal volunteer subjects (Table 3).

TLR Activation of Whole Blood Cell Cultures: Whole blood cultures were prepared and stimulated with zymosan to invoke TLR2-mediated responses or stimulated with LPS to invoke TLR4-mediated responses. The results in Fig. 1 show the ability of TLR2 and TLR4 activation to stimulate the production of TNF $\alpha$. TNF $\alpha$ production in the absence of stimulation (baseline) was marginal. Activation of TLR2 induced marked production of TNF $\alpha$ in cultures from both controls (healthy volunteers) and subjects. TNF $\alpha$ 
production by cultures from the subjects was suppressed as it was only $~ 25 \%$ of that observed in control whole blood cell cultures. TLR4 evoked responses were similar to that of TLR2.

The data presented in Fig. 2 show the ability of TLR activation to induce IL-1 $\beta$ production. TLR2 activation with zymosan induced significant levels of IL-1 $\beta$ in both the control population and subjects. IL-1 $\beta$ production in the subjects was reduced by $\sim 50 \%$ in the subjects as compared with controls. TLR4 activation with LPS also induced significant levels of IL-1 $\beta$; however, there was no difference between controls and subjects.

The strongest response to the TLR agonists was for IL-6 (Fig. 3). Significant levels of IL6 were seen in the unstimulated (baseline) cultures from subjects $(\sim 250 \mathrm{pg} / \mathrm{mL})$. Similar to other cytokines TLR2 and TLR4 activation stimulated the production of high levels of IL-6. In contrast with IL-1 $\beta$ and TNF $\alpha$, TLR4 activation induced over 2-fold greater levels of IL-6 than TLR2 activation. Whole blood cell cultures from controls were markedly more responsive to zymosan or LPS with up to 3-fold greater levels of IL-6 than subject cultures.

IL-10 levels in the whole blood cell cultures were relatively low, with only TLR4 activation inducing significant increases over that observed at baseline (Fig. 4). As seen with the other cytokines TLR4 activation of IL-10 production was significantly less in the subjects as compared with controls. 


\section{DISCUSSION}

The ability of the innate immune system to respond to exogenous and endogenous signals is crucial to the maintenance of a healthy response to infection or tissue damage. A response that is too robust or one that is inadequate can result in an imbalance in immunity and lead to a dysfunctional recovery from pathogen or injury [4;5;21]. Since the discovery of toll-like receptors (TLRs), a family of pattern-recognition receptors (PRRs) the understanding of innate immunity has been greatly improved [22]. TLRs have been implicated in the immune regulation of various disease conditions including allergies, autoimmunity, atherosclerosis, burn and sepsis [22]. In this study, we sought to characterize the TLR-mediated immunoinflammatory response in critically injured subjects. To this end, we obtained whole blood from severely injured trauma patients, stimulated it with TLR2 and TLR4 agonists, and measured the inflammatory response via representative pro- and anti-inflammatory cytokines and compared the resulting cytokine profile to healthy uninjured subjects. Due to the nature of trauma, it is impossible to predict admission and enroll the injured subjects prior to injury and inflammatory disturbances. For this reason, the healthy volunteers served as surrogates in order to estimate the available response in a normal human population before injury.

Cytokine levels in the peripheral circulation can serve as markers of systemic illness; however, in the study herein only IL-6 was elevated in the injured population. This is consistent with previous studies showing elevated IL-6 levels in trauma patients [23;24]. The lack of a measurable IL-1 $\beta$, TNF $\alpha$ or IL-10 in the plasma may be inpart due to the time of sample collection, which was approximately 1 week post-injury. 
The production of pro-inflammatory cytokines in response to TLR2 or TLR4 agonists in the subject population was notable. Zymosan and LPS stimulation of TLR2 and TLR4, respectively resulted in greater induction of pro-inflammatory cytokines (TNF $\alpha$, IL-1 $\beta$, and IL-6) than anti-inflammatory IL-10. This is relevant given that a pro-inflammatory response is important in the setting of secondary infection or subsequent injury following the initial trauma, such as surgery [25-28]. The subject samples in the study herein were collected around 7 days after admission to the ICU; this is within the time period when the patient is at risk for nosocomial infection from indwelling lines, tubes, and catheters [29;30]. Our previous work has shown in this patient cohort that those with pulmonary complications exhibited a significant Th-17 response in the lung and blood, suggesting that this pro-inflammatory milieu may be a contributing factor to such complications [18].

In contrast, the pro-inflammatory response seen in cells from healthy volunteers was much more robust than that observed in subjects. The measured levels of TNF $\alpha$, IL-1 $\beta$, and IL-6 are all significantly higher in controls versus the study population. This demonstrates that the peripheral blood cells of healthy, uninjured subjects are more responsive to TLR agonists than cells from injured patients in the ICU. The suppressed pro-inflammatory response is indicative of impairment in the immune system following trauma [31;32]. Heagy et al. [19] demonstrated that under similar conditions as employed herein that ICU patients with suppressed LPS-stimulated cytokine release capacity had significantly poorer clinical outcomes. They concluded that the use of the whole blood stimulation assay might be useful to identify ICU patients with severe 
sepsis. This finding may also signify that pre-injury cytokine stores or the ability to engineer these inflammatory cytokines has not been depleted by an initial insult of injury. Conversely, this may signify that the response to these TLR agonists is blunted after an initial injury, possibly through a loss of receptor expression or uncoupling. Regardless of the mechanism, the data suggest that that severely injured patients are less able to respond to insult via a TLR-mediated process.

We found that the production of the anti-inflammatory cytokine, IL-10, was not statistically different at baseline or in response toTLR2 activation when comparing the two populations. However, the control population elaborated a greater IL-10 response than the injured population when stimulated with the TLR4 agonist, LPS. This mimics the pro-inflammatory cytokine response and may also be reflective of a deficiency in the ability to produce a general cytokine response. The trend towards a higher IL-10 response at baseline in the subjects may represent the compensatory inflammatory response (CARS) which acts to deactivate the immune system tasked and restore homeostasis from the initial post-injury inflammatory state [33].

TLRs can regulate inflammatory responses to various endogenous stimuli that include heat-shock proteins, extracellular matrix breakdown products and necrotic cells. These stimuli are collectively known as damage-associated molecular patterns (DAMPs) [34]. A relevant model of tissue injury (or trauma) has been developed by Matzinger which involves danger signaling [35]. In this “Danger" model, the immunoinflammatory response initiated by injury is the result of the recognition of molecular patterns by cell 
receptors, such as TLRs that are associated with either pathogens or certain cellular components released by damaged cells (i.e., DAMPs). Importantly, this model reconciles the paradox of the similar inflammatory response observed under sterile and non-sterile conditions. Seminal findings by Zhang et al. [10] demonstrated that trauma causes tissue injury that releases mitochondrial DAMPs capable of activating neutrophils via TLRs and elicit neutrophil-mediated organ injury. While the study herein did not assess DAMPs it is interesting to speculate that elevated DAMP levels in our trauma patient cohort affected TLR-induced cytokine production in our in vitro assays.

There are several deficiencies, if addressed which would strengthen this study. First of all, the time from injury until enrollment and obtaining a blood sample is variable. This may result in more heterogeneity in the injured population. Secondly, the healthy volunteers may not be representative of the injured individuals at baseline prior to injury. Additionally, the possibility exists that there are inherent differences in these groups which account for the differences in response to TLR agonists. This is unlikely as the addition of more control subject samples to this population would strengthen the conclusions produced. Lastly, we have not included data that examines TLR2 and TLR4 expression on cell surfaces; however Ono et al. [36] have shown that there is no change in TLR2 or TLR4 expression after surgery, suggesting that there may not be alterations in expression in our study. This information could possibly provide mechanistic data explaining the decreased cytokine response in the injured population. This data is currently being collected and examined in ongoing studies in our laboratory. 


\section{CONCLUSIONS}

Based on the data presented, we conclude that both healthy volunteers and severely injured trauma subjects produce inflammatory cytokines in response to in-vitro TLR2 and TLR4 agonists. However, the severely injured patients were unable to elaborate as robust of a response to TLR stimulation as compared to the healthy control population. Overall, TLR-mediated activation of circulating leukocytes from trauma ICU subjects is suppressed, which may influence the host defense system and alter susceptibility to inflammatory and infectious complications. 


\section{ACKNOWLEDGEMENTS}

Travis L Holloway, MD is supported under the UTHSCSA Department of Surgery NIH T32 Training Grant NIH Grant (5T32GM079085) and the project was in part supported by NIH/NCRR UL 1RR025767, NIH-NCI P30 CA54174 and UL1RR025767. These finding were presented in part at the 36th annual meeting of the Shock Society in San Diego CA. The authors wish to acknowledge the excellent technical assistance of Teresa Craig and Qiong Zhang. The opinions or assertions contained herein are the private views of the author and are not to be construed as official or as reflecting the views of the Department of the Army or the Department of Defense. 


\section{REFERENCES}

1. Shere-Wolfe, R. F., Galvagno, S. M., Jr., and Grissom, T. E. Critical care considerations in the management of the trauma patient following initial resuscitation. Scand. J Trauma Resusc. Emerg. Med 20:68, 2012.

2. Trouillet, J. L., Chastre, J., Vuagnat, A., Joly-Guillou, M. L., Combaux, D., Dombret, M. C., and Gibert, C. Ventilator-associated pneumonia caused by potentially drug-resistant bacteria. Am. J Respir. Crit Care Med 157:531-539, 1998.

3. Dancey, D. R., Hayes, J., Gomez, M., Schouten, D., Fish, J., Peters, W., Slutsky, A. S., and Stewart, T. E. ARDS in patients with thermal injury. Intensive Care Med 25:1231-1236, 1999.

4. Baue, A. E., Durham, R., and Faist, E. Systemic inflammatory response syndrome (SIRS), multiple organ dysfunction syndrome (MODS), multiple organ failure (MOF): are we winning the battle? Shock 10:79-89, 1998.

5. Manson, J., Thiemermann, C., and Brohi, K. Trauma alarmins as activators of damage-induced inflammation. Br. J Surg. 99 Suppl 1:12-20, 2012.

6. Kawai, T., and Akira, S. The role of pattern-recognition receptors in innate immunity: update on Toll-like receptors. Nat. Immunol 11:373-384, 2010.

7. Janeway, C. A., Jr., and Medzhitov, R. Innate immune recognition. Annu. Rev. Immunol. 20:197-216, 2002.

8. Miyake, K. Innate immune sensing of pathogens and danger signals by cell surface Toll-like receptors. Semin. Immunol. 19:3-10, 2007.

9. Drage, M. G., Pecora, N. D., Hise, A. G., Febbraio, M., Silverstein, R. L., Golenbock, D. T., Boom, W. H., and Harding, C. V. TLR2 and its co-receptors determine responses of macrophages and dendritic cells to lipoproteins of Mycobacterium tuberculosis. Cell Immunol. 258:29-37, 2009.

10. Zhang, Q., Raoof, M., Chen, Y., Sumi, Y., Sursal, T., Junger, W., Brohi, K., Itagaki, K., and Hauser, C. J. Circulating mitochondrial DAMPs cause inflammatory responses to injury. Nature 464:104-107, 2010.

11. Schwacha, M. G., Rani, M., Zhang, Q., Nunez-Cantu, O., and Cap, A. P. Mitochondrial damage-associated molecular patterns activate gammadelta T-cells. Innate. Immun. 20:261-268, 2014.

12. Krysko, D. V., Agostinis, P., Krysko, O., Garg, A. D., Bachert, C., Lambrecht, B. N., and Vandenabeele, P. Emerging role of damage-associated molecular patterns derived from mitochondria in inflammation. Trends Immunol. 32:157-164, 2011. 
13. Darwiche, S. S., Ruan, X., Hoffman, M. K., Zettel, K. R., Tracy, A. P., Schroeder, L. M., Cai, C., Hoffman, R. A., Scott, M. J., Pape, H. C., and Billiar, T. R. Selective roles for toll-like receptors 2,4 , and 9 in systemic inflammation and immune dysfunction following peripheral tissue injury. J Trauma Acute. Care Surg. 74:1454-1461, 2013.

14. West, A. P., Koblansky, A. A., and Ghosh, S. Recognition and signaling by toll-like receptors. Annu. Rev. Cell Dev. Biol. 22:409-437, 2006.

15. Foell, D., Wittkowski, H., and Roth, J. Mechanisms of disease: a 'DAMP' view of inflammatory arthritis. Nat. Clin. Pract. Rheumatol. 3:382-390, 2007.

16. Chen, X. L., Sun, L., Guo, F., Wang, F., Liu, S., Liang, X., Wang, R. S., Wang, Y. J., and Sun, Y. X. High-mobility group box-1 induces proinflammatory cytokines production of Kupffer cells through TLRs-dependent signaling pathway after burn injury. PLoS. One. 7:e50668, 2012.

17. Ranieri, V. M., Rubenfeld, G. D., Thompson, B. T., Ferguson, N. D., Caldwell, E., Fan, E., Camporota, L., and Slutsky, A. S. Acute respiratory distress syndrome: the Berlin Definition. JAMA 307:2526-2533, 2012.

18. Holloway, T. L., Rani, M., Cap, A. P., Stewart, R. M., and Schwacha, M. G. The association between the Th-17 immune response and pulmonary complications in a trauma ICU population. Cytokine 76:328-333, 2015.

19. Heagy, W., Nieman, K., Hansen, C., Cohen, M., Danielson, D., and West, M. A. Lower levels of whole blood LPS-stimulated cytokine release are associated with poorer clinical outcomes in surgical ICU patients. Surg. Infect. (Larchmt. ) 4:171180, 2003.

20. Schwacha, M. G., Zhang, Q., Rani, M., Craig, T., and Oppeltz, R. F. Burn enhances Toll-like receptor induced responses by circulating leukocytes. Int J Clin Exp Med 5:136-144, 2012.

21. Partrick, D. A., Moore, E. E., Moore, F. A., Biffl, W. L., and Barnett, C. C., Jr. Release of anti-inflammatory mediators after major torso trauma correlates with the development of postinjury multiple organ failure. Am. J Surg. 178:564-569, 1999.

22. Cook, D. N., Pisetsky, D. S., and Schwartz, D. A. Toll-like receptors in the pathogenesis of human disease. Nat. Immunol. 5:975-979, 2004.

23. Guisasola, M. C., Ortiz, A., Chana, F., Alonso, B., and Vaquero, J. Early inflammatory response in polytraumatized patients: Cytokines and heat shock proteins. A pilot study. Orthop. Traumatol. Surg. Res. 2015.

24. Wang, Y. X., Xu, X. Y., Su, W. L., Wang, Q., Zhu, W. X., Chen, F., Jin, G., Liu, Y. J., Li, Y. D., Sun, Y. P., Gao, W. C., and Ruan, C. P. Activation and clinical 
significance of p38 MAPK signaling pathway in patients with severe trauma. $J$ Surg. Res. 161:119-125, 2010.

25. Dobson, G. P. Addressing the Global Burden of Trauma in Major Surgery. Front Surg. 2:43, 2015.

26. Headland, S. E., and Norling, L. V. The resolution of inflammation: Principles and challenges. Semin. Immunol 27:149-160, 2015.

27. Portou, M. J., Baker, D., Abraham, D., and Tsui, J. The innate immune system, tolllike receptors and dermal wound healing: A review. Vascul. Pharmacol. 71:31-36, 2015.

28. Evans, C., Galustian, C., Kumar, D., Hagger, R., Melville, D. M., Bodman-Smith, M., Jourdan, I., Gudgeon, A. M., and Dalgleish, A. G. Impact of surgery on immunologic function: comparison between minimally invasive techniques and conventional laparotomy for surgical resection of colorectal tumors. Am. J Surg. 197:238-245, 2009.

29. Duane, T. M., Kikhia, R. M., Wolfe, L. G., Ober, J., and Tessier, J. M. Understanding Gram-negative Central Line-Associated Blood Stream Infection in a Surgical Trauma ICU. Am. Surg. 81:816-819, 2015.

30. Ong, D. S., Bonten, M. J., Safdari, K., Spitoni, C., Frencken, J. F., Witteveen, E., Horn, J., Klein Klouwenberg, P. M., and Cremer, O. L. Epidemiology, Management, and Risk-Adjusted Mortality of ICU-Acquired Enterococcal Bacteremia. Clin Infect. Dis. 61:1413-1420, 2015.

31. Bone, R. C. Sir Isaac Newton, sepsis, SIRS, and CARS. Crit Care Med 24:11251128, 1996.

32. Binkowska, A. M., Michalak, G., and Slotwinski, R. Current views on the mechanisms of immune responses to trauma and infection. Cent. Eur. J Immunol 40:206-216, 2015.

33. Ward, N. S., Casserly, B., and Ayala, A. The compensatory anti-inflammatory response syndrome (CARS) in critically ill patients. Clin Chest Med 29:617-25, viii, 2008.

34. Beg, A. A. Endogenous ligands of Toll-like receptors: implications for regulating inflammatory and immune responses. Trends Immunol. 23:509-512, 2002.

35. Matzinger, P. The danger model: a renewed sense of self. Science 296:301-305, 2002.

36. Ono, S., Tsujimoto, H., Hiraki, S., Takahata, R., Kinoshita, M., and Mochizuki, H. Sex differences in cytokine production and surface antigen expression of peripheral blood mononuclear cells after surgery. Am. J Surg. 190:439-444, 2005. 


\section{TABLES}

Table 1. Injuries for inclusion in the study ${ }^{\mathrm{a}}$

\begin{tabular}{llc}
\hline Region & Inclusion Injuries & AIS \\
\hline Neck & Cervical spine fracture with persistent neurologic deficit & $4+$ \\
Face & Lefort III facial fracture & $4+$ \\
Lung & Pulmonary contusion in any location & $3+$ \\
Chest & Rib fracture >3 in any location & $3+$ \\
Heart & Blunt cardiac injury with cardiac failure/valve & $4+$ \\
Vascular & involvement/septal rupture & $4+$ \\
Abdomen & Gortic laceration/intimal tear & $3+$ \\
Pelvis & Orade 3 or greater liver, spleen, kidney, pancreas injury & $3+$ \\
Extremity & Open or displaced long bone fracture any location & $3+$ \\
Extremity & Amputation above fingers/toes & $3+$ \\
\hline
\end{tabular}

${ }^{a}$ Injury enrollment criteria consisted of Abbreviated Injury Scores (AISs) of 3 or greater, representing severe injury. Individuals with head injuries were enrolled, but head injury alone (in the absence of another severe injury) was not sufficient for enrollment. 
Table 2. Demographics ${ }^{\mathrm{a}}$

\begin{tabular}{lc}
\hline Age (years) & $40.7 \pm 19.7$ \\
Gender (\% male) & 86 \\
Ethnicity (\% caucasian) & $57 \%$ \\
ISS & $37.6 \pm 18.8$ \\
Hospital LOS (days) & $20.6 \pm 9.8$ \\
ICU LOS (days) & $6.6 \pm 4.2$ \\
Admission time until sample collection & \\
(days) & $3.3 \pm 2.7$ \\
Previous transfusion (days prior) & \\
& $2.3 \pm 0.9$ \\
\hline
\end{tabular}

${ }^{\mathrm{a}}$ The demographics for all 21 enrolled subjects. ISS=Injury Severity Score LOS=Length of Stay. Data are expressed as mean \pm SD. 
Table 3. Plasma cytokine levels $(\mathrm{pg} / \mathrm{mL})^{\mathrm{a}}$

\begin{tabular}{lcc}
\hline & Controls $(\mathrm{n}=6)$ & Subjects $(\mathrm{n}=21)$ \\
\hline TNF $\alpha$ & $1 \pm 1^{\mathrm{b}}$ & $8 \pm 4$ \\
IL-1 $\beta$ & n.d. $^{\mathrm{c}}$ & $1 \pm 1$ \\
IL-6 & $3 \pm 1$ & $271 \pm 146^{\mathrm{d}}$ \\
IL-10 & $2 \pm 1$ & $4 \pm 1$ \\
\hline
\end{tabular}

a. Cytokine levels were determined in plasma samples as described in the materials and methods section.

b. Data are mean \pm SEM

c. n.d. = not detectable

d. $\mathrm{p}<0.05$ as compared with respective control 


\section{FIGURE LEGENDS}

Figure 1. TLR-induced TNF $\alpha$ production. Whole blood was collected from trauma patients (subjects; $n=21$ ) and healthy volunteers (controls; n=6) and cultured for $3 \mathrm{hrs}$ with zymosan (TLR2 agonist), LPS (TLR4 agonist) or left unstimulated (baseline) as described in the materials and methods. Supernatant content of TNF $\alpha$ was determined by Bioplex assay. Data are the mean \pm SEM for $n=6-21$ /group. $* p<0.05$ as compared with baseline $\uparrow p<0.05$ as compared with respective control.

Figure 2. TLR-induced IL-1 $\beta$ production. Whole blood was collected from trauma patients (subjects; $n=21$ ) and healthy volunteers (controls; $n=6$ ) and cultured for $3 \mathrm{hrs}$ with zymosan (TLR2 agonist), LPS (TLR4 agonist) or left unstimulated (baseline) as described in the materials and methods. Supernatant content of IL-1 $\beta$ was determined by Bioplex assay. Data are the mean \pm SEM for $n=6-21$ /group. ${ }^{*} p<0.05$ as compared with baseline. $\dagger p<0.05$ as compared with respective control.

Figure 3. TLR-induced IL-6 production. Whole blood was collected from trauma patients (subjects; $n=21$ ) and healthy volunteers (controls; $n=6$ ) and cultured for 3 hrs with zymosan (TLR2 agonist), LPS (TLR4 agonist) or left unstimulated (baseline) as described in the materials and methods. Supernatant content of IL-6 was determined by Bioplex assay. Data are the mean \pm SEM for $n=6-21$ /group. $* p<0.05$ as compared with baseline $\uparrow p<0.05$ as compared with respective control. $\ddagger p<0.05$ as compared with respective TLR2 value. 
Figure 4. TLR-induced IL-10 production. Whole blood was collected from trauma patients (subjects; $\mathrm{n}=21$ ) and healthy volunteers (controls; $\mathrm{n}=6$ ) and cultured for $3 \mathrm{hrs}$ with zymosan (TLR2 agonist), LPS (TLR4 agonist) or left unstimulated (baseline) as described in the materials and methods. Supernatant content of IL-10 was determined by Bioplex assay. Data are the mean \pm SEM for $\mathrm{n}=6$-21/group. ${ }^{*} p<0.05$ as compared with baseline $\uparrow p<0.05$ as compared with respective control. 


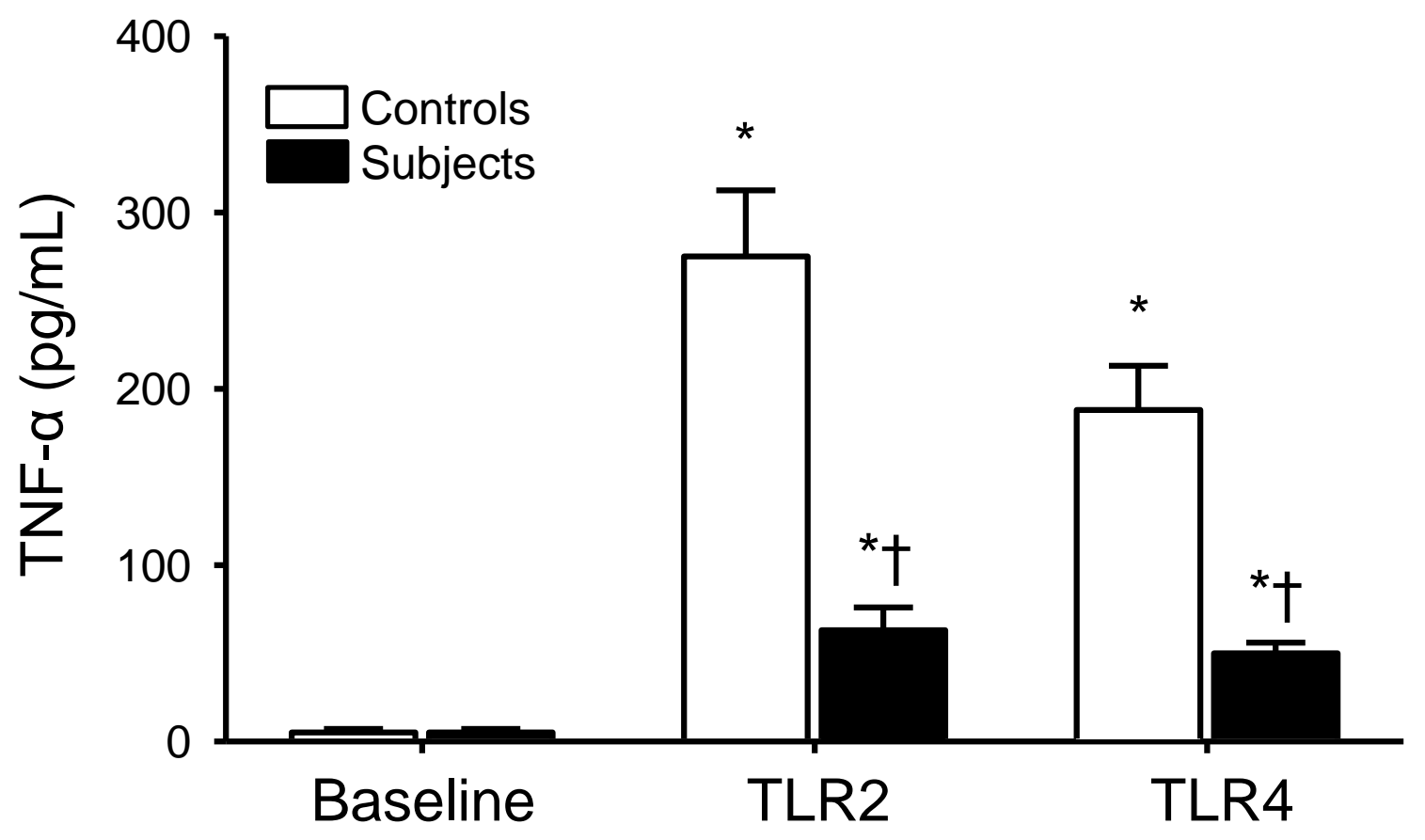




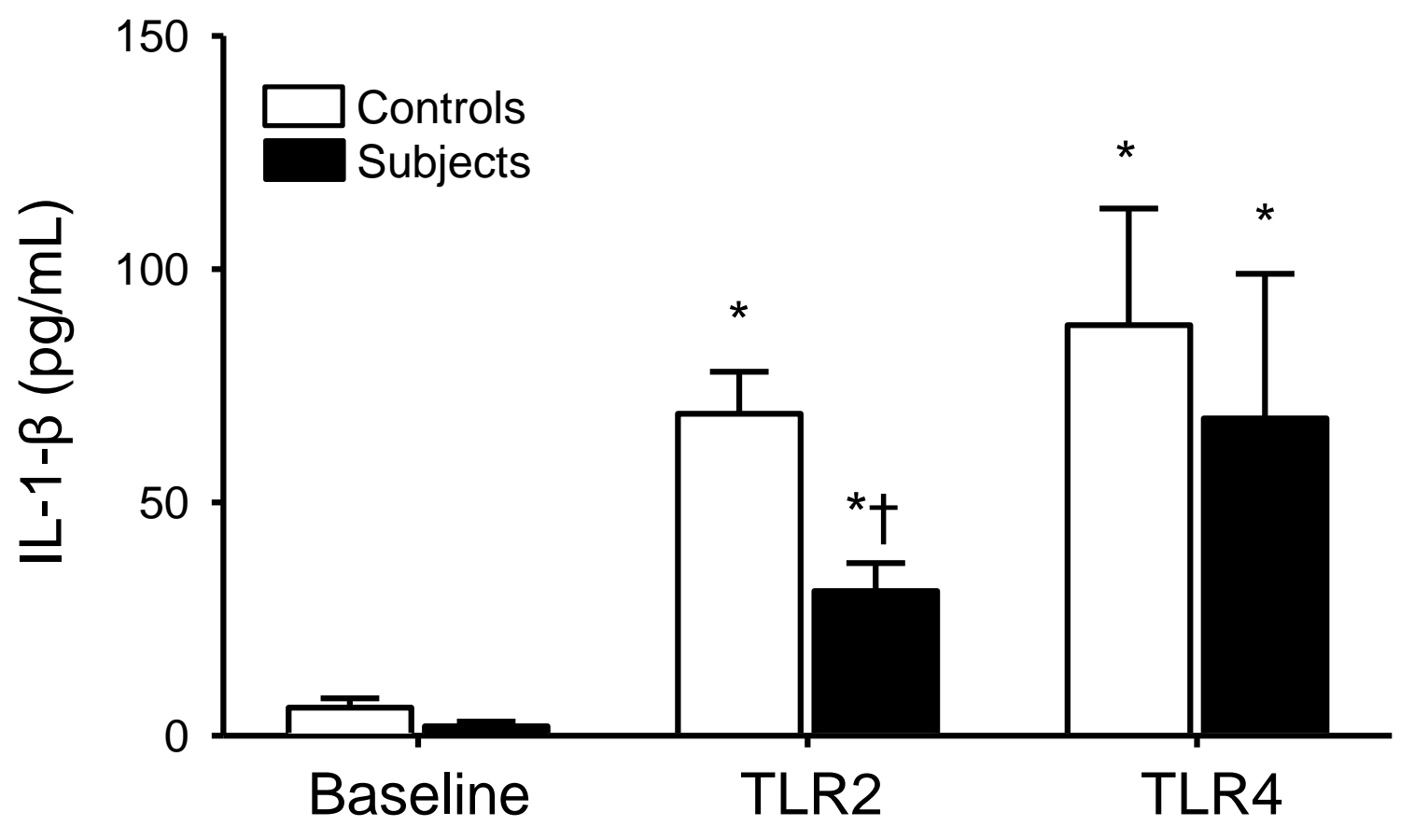




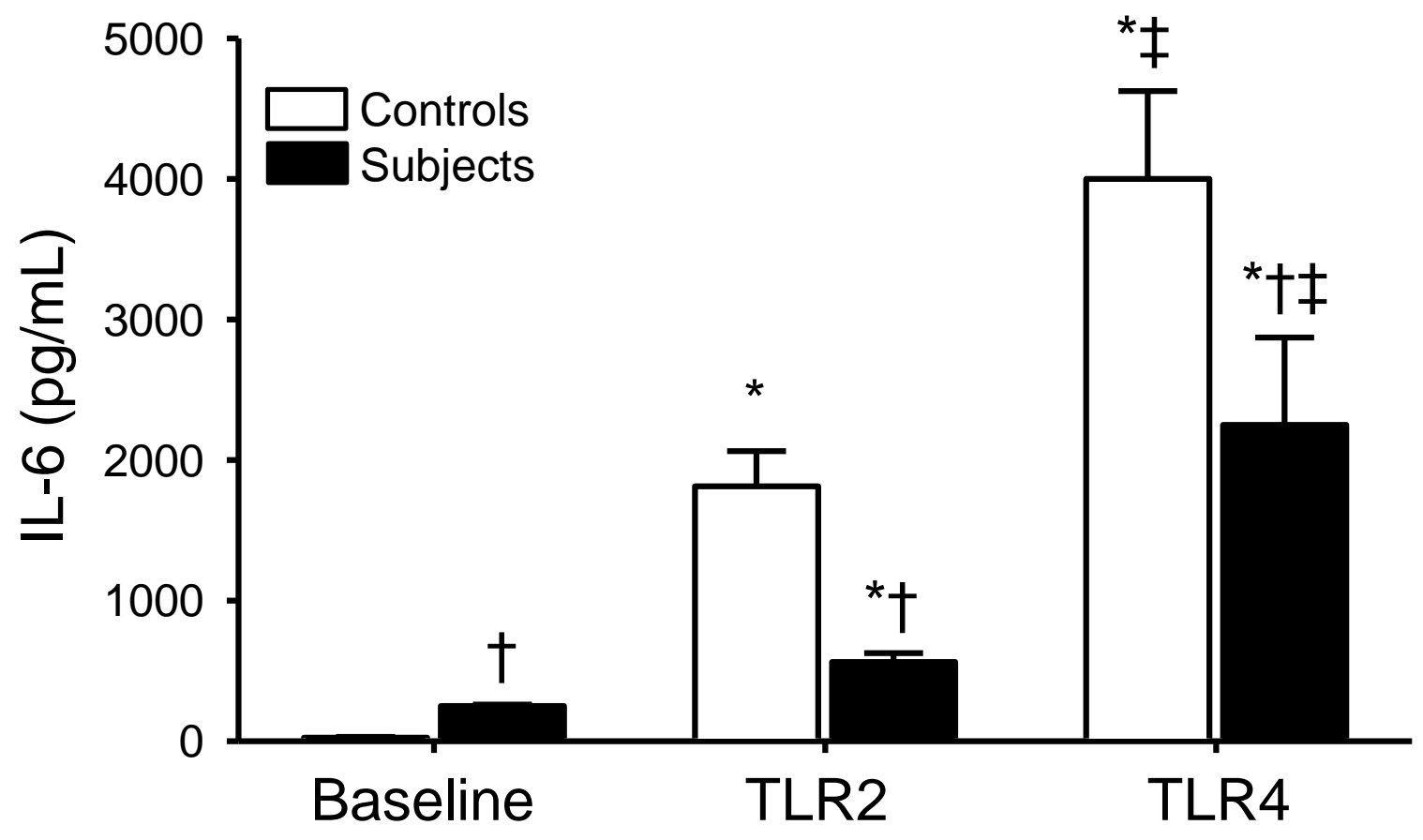




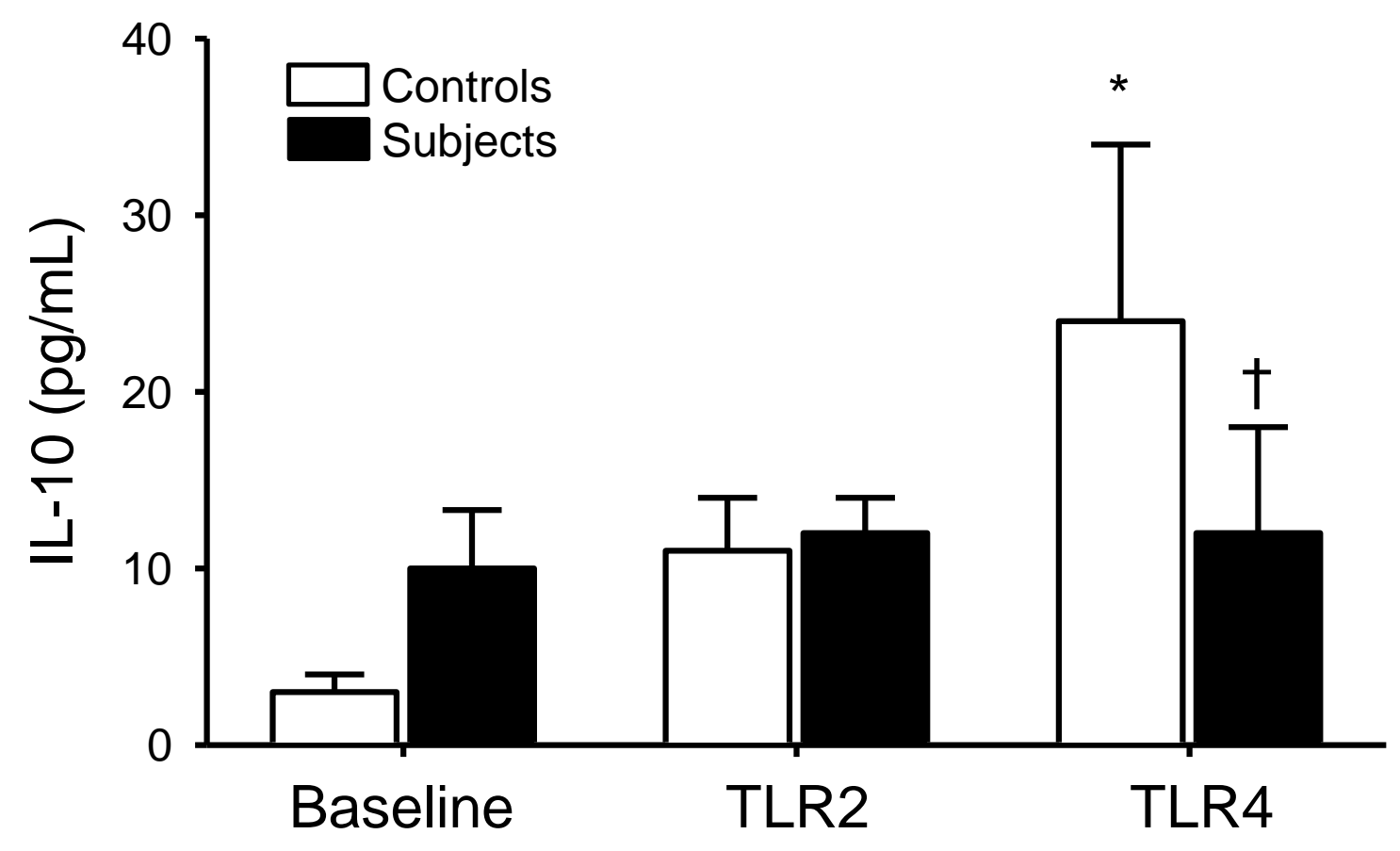

